\title{
Contamination des basidiomycètes (Volvariella volvacea et Termitomyces spp) des marchés abidjanais par le plomb, le cadmium, le mercure et le zinc
}

\author{
Gains Kouakou KPAN KPAN ${ }^{1 *}$, Lazare Brou $\mathrm{YAO}^{2}$, Ardjouma DEMBELE ${ }^{2}$, \\ Sory Karim TRAORE ${ }^{3}$ et Francis MESSOUM ${ }^{4}$ \\ ${ }^{1}$ Laboratoire de Biologie et Cytologie Animale, UFR des Sciences de la Nature Université Nangui Abrogoua, \\ 02 BP 801 Abidjan 02, Côte d'Ivoire. \\ ${ }^{2}$ Laboratoire d'Appui au Développement Agricole (LANADA), Laboratoire Central d'Agrochimie et \\ d'Ecotoxicologie (LCAE) 04 BP 612 Abidjan 04, Côte d'Ivoire. \\ ${ }^{3}$ Laboratoire de Chimie Santé et Environnement, UFR des Sciences et Gestion de l'Environnement, \\ Université Nangui Abrougoua, 02 BP 801 Abidjan 02, Côte d'Ivoire. \\ ${ }^{4}$ Direction de la Recherche Scientifique du Ministère de l'Enseignement Supérieur et de la Recherche \\ Scientifique, Côte d'Ivoire. \\ *Auteur correspondant ; E-mail : oraclegains@gmail.com ; Tel. : (+225) 47663894
}

\section{RESUME}

Le monitoring des éléments traces métalliques (ETM) de l'environnement et des réseaux trophiques a considérablement gagné du terrain durant ces dernières décennies afin de prévenir les problèmes environnementaux et de santé publique. Cette étude s'inscrit dans ce contexte de monitoring et entend évaluer le niveau de contamination de Volvariella volvacea, Termitomyces robustus et Termitomyces letestui par le plomb, le cadmium, le mercure et le zinc. Le dosage des ETM s'effectué par spectrométrie d'absorption atomique sur 77 échantillons de champignon issus des marchés abidjanais. La teneur minimale du plomb, du cadmium et du mercure est $0,01 \pm 0,01 \mathrm{mg} / \mathrm{kg}$ et celle du zinc est $0,001 \pm 0,001 \mathrm{mg} / \mathrm{kg}$. La teneur maximale du plomb est $1,11 \pm 0,01 \mathrm{mg} / \mathrm{kg}$ et a été détectée chez Termitomyces letestui. La plus grande teneur du cadmium est $0,52 \pm 0,02 \mathrm{mg} / \mathrm{kg}$ et a été détectée chez Termitomyces robustus. Les teneurs maximales du mercure et du zinc sont respectivement $0,85 \pm 0,04 \mathrm{mg} / \mathrm{kg}$ chez Termitomyces letestui et $0,07 \pm 0,03 \mathrm{mg} / \mathrm{kg}$ chez Termitomyces robustus. Les teneurs moyennes métalliques oscillent entre $0,003 \pm 0,001 \mathrm{mg} / \mathrm{kg}$ (zinc) et $0,30 \pm$ $0,05 \mathrm{mg} / \mathrm{kg}$ (plomb). Les teneurs moyennes métalliques de ces champignons sont conformes aux standards (CE) nº 1881/2006 de l'Union Européenne.

(C) 2014 International Formulae Group. All rights reserved.

Mots clés : Elément trace métallique, carpophore, bioaccumulation.

\section{INTRODUCTION}

Depuis plusieurs décennies, les métaux font partie du quotidien de l'homme et leur usage a permis à l'homme d'acquérir d'énormes progrès technologiques tels que la construction automobile, la production d'énergie, la balistique, l'architecture etc. Pour satisfaire la demande en métaux, un accent particulier a été mis sur l'exploitation des gisements métallifères et la métallurgie. L'utilisation accrue des métaux a engendré la contamination des compartiments de la 
biosphère (air, eau, sol) et des chaînes trophiques par les éléments traces métalliques (ETM) (Nassif et Saade, 2010; Shivakumar et al., 2012).

La contamination du réseau trophique par les éléments traces métalliques est une situation délicate qui est souvent source de problèmes de santé publique. En effet, à cause de leur caractère non biodégradable, ces métaux sont capables de s'accumuler dans les tissus mous et les os des hommes et des animaux. Il résulte de cette accumulation des graves affections, notamment la protéinurie cadmique, l'ostéomalacie, l'hydrargyrisme, la néphrite tubulaire et les troubles de la croissance chez l'enfant (Unak et al., 2007 ; Musa et Abdullahi, 2013)

$\mathrm{Vu}$, les problèmes de santé imputables aux éléments traces métalliques et la circulation permanentes de ces derniers dans le réseau trophique, on assiste de plus en plus à une émergence de programmes de monitoring des différentes composantes (flore, faune, eau, air et sol) des écosystèmes dans l'optique de garantir à l'homme un environnement sain et une sécurité alimentaire. Parmi les matrices ciblées par la recherche, les champignons basidiomycètes comestibles occupent une place de choix pour deux raisons essentiellement à savoir d'une part le pouvoir de bioaccumulation des éléments traces métalliques de ces champignons (Radulescu et al., 2010); et d'autre part les effets bénéfiques de cette denrée alimentaire sur la santé (Györfi et al., 2010 ; Mujic et al., 2011 ; Athanasakis et al., 2013).

Malgré ce caractère ambivalent des champignons comestibles, les données officielles mettant en relief les champignons sauvages comestibles de Côte d'Ivoire et les éléments traces métalliques sont quasi inexistantes. Ce manque de données relatives à la teneur des éléments traces métalliques des basidiomycètes ivoiriens peut pousser certains consommateurs à abandonner cette denrée ; ce qui priverait ces derniers des effets bénéfiques des champignons. D'autres par contre continueraient à consommer les champignons sans précaution, s'exposant ainsi aux effets toxiques des éléments traces métalliques.

Pour la sécurité sanitaire des consommateurs de champignon en Côte d'Ivoire et la préservation de la qualité de l'environnement, le dosage des éléments traces métalliques des champignons à carpophore comestible s'avère alors très capital. C'est dans ce contexte que s'inscrit la présente étude dont l'objectif est de connaître le niveau de concentration des éléments traces métalliques (plomb, cadmium, mercure et zinc) de Volvariella volvacea et de deux espèces de Termitomyces spp.

\section{MATERIEL ET METHODES \\ Echantillonnage et préparation des échantillons}

Les champignons utilisés dans cette étude sont Volvariella volvacea communément appelé champignon des palmiers, Termitomyces letestui et Termitomyces robustus. Ces espèces de champignons ont été achetées chez des vendeuses de différentes communes, notamment les communes de Port-Bouët, Koumassi, Adjamé, Abobo et Yopougon. La taille de chaque échantillon (200 g) a été mesurée à l'aide d'une balance électronique SHIMADZU avec une précision de $\pm 0,1 \mathrm{~g}$. $\mathrm{Au}$ total, 77 échantillons dont 30 pour chacune des espèces Volvariella volvacea et Termitomyces lestui et 17 pour Termitomyces robustus ont été collectés.

Tous les échantillons ont été découpés en petits morceaux au moyen d'un couteau muni d'une lame en acier inoxydable, puis broyés en fines particules à l'aide d'un mixeur Ultra Turax dotée d'une lame en acier inoxydable. Les broyats obtenus ont été conservés dans des sachets (stomachers) à la température de $-27^{\circ} \mathrm{C}$.

\section{Digestion et minéralisation des échantillons \\ La digestion et la minéralisation des} échantillons ont été réalisées au Laboratoire Central pour l'Hygiène Alimentaire et de 
l'Agro-Industrie (LCHAI) suivant les méthodes AOAC (Association of Official Analytical Chemists). Pour la détermination du plomb, du cadmium et du zinc, la méthode officielle AOAC $n^{\circ} 999.10$ a été utilisée (AOAC, 2003). Quant au mercure, la méthode officielle AOAC $n^{\circ} 977.13$ a été adoptée (AOAC, 1978). Ces différentes méthodes ont été adaptées aux conditions du laboratoire afin d'optimiser les résultats.

Pour digérer chaque échantillon, une prise d'essai de $0,5 \mathrm{~g}$ de broyat a été placée dans un tube en téflon. Sous une hotte, $5 \mathrm{ml}$ d'acide nitrique $\left(\mathrm{HNO}_{3}\right)$ à $65 \%, 2 \mathrm{ml}$ d'acide sulfurique $\left(\mathrm{H}_{2} \mathrm{SO}_{4}\right)$ à $98 \%$ et $1 \mathrm{ml}$ de peroxyde d'hydrogène $\left(\mathrm{H}_{2} \mathrm{O}_{2}\right)$ à $30 \%$ ont été successivement ajouté au broyat. Un autre tube en téflon contenant de l'eau bidistillée a été utilisé comme blanc. A ce tube (blanc) les mêmes réactifs qui ont servi à la digestion de la matrice ont été ajoutés dans le même ordre. Les téflons ont été conservés environ 5 minutes sous la hotte afin que celle-ci aspire la fumée dégagée par la réaction. Ensuite, les téflons ont été montés sur un support puis placés dans un four à micro-onde Milestone de type DG-AG-03 pour la minéralisation de l'échantillon. La minéralisation a duré 30 minutes, puis les tubes en téflon ont été retirés et déposés sur une paillasse pour leur refroidissement après lequel le contenu de chaque tube en téflon a été renversé dans un tube à essai en verre puis complété à $25 \mathrm{ml}$ avec de l'eau bidistillée. Le minéralisât a été ensuite homogénéisé au moyen d'un agitateur rotatif (Vortex).

\section{Quantification des éléments traces métalliques}

La détection des éléments traces métalliques s'est faite à l'aide d'un spectromètre d'absorption atomique SPECTRA 110 (VARIAN) à partir duquel un étalonnage a été préalablement réalisé pour chaque métal avec les concentrations croissantes de $10 \mu \mathrm{g} / \mathrm{l}, 20 \mu \mathrm{g} / \mathrm{l}, 30 \mu \mathrm{g} / \mathrm{l}$ et 40 $\mu \mathrm{g} / \mathrm{l}$. Les coefficients de corrélation $\left(\mathrm{R}^{2}\right)$ entre les absorbances et les concentrations métalliques des étalons, se situent entre 0,997 et 0,999 (Figure 1)

Les teneurs métalliques données par le spectromètre sont exprimées $\mu \mathrm{g} / \mathrm{l}$. Les teneurs métalliques en $\mathrm{mg} / \mathrm{kg}$ (ppm) ont été obtenues à partir des concentrations lues sur le spectromètre, concentrations auxquelles a été appliquée la formule suivante:

$$
C_{m}=\frac{\left[\left(c_{l}-c_{b}\right) 25 f\right]}{M}
$$

$\mathrm{C}_{\mathrm{m}}$ : Concentration en partie par million $(\mathrm{mg} / \mathrm{kg})$;

$\mathrm{C}_{1}$ : Concentration donnée par l'appareil $(\mathrm{mg} / \mathrm{l})$;

$\mathrm{C}_{\mathrm{b}}$ : Concentration du blanc $(\mathrm{mg} / \mathrm{l})$;

f : Facteur de dilution;

M : Masse de matrice minéralisée (g).

\section{Evaluation du niveau de contamination des champignons}

Pour évaluer le niveau contamination des espèces de champignon, les teneurs métalliques moyennes sont comparées entre elles dans un premier temps, puis dans un second temps aux normes officielles de l'Union Européenne (UE). Selon le règlement (CE) $n^{\circ} 1881 / 2006$, les limites maximales de résidu (LMR) du plomb et du cadmium des champignons comestibles, sont respectivement $0,3 \mathrm{mg} / \mathrm{kg}$ et $0,2 \mathrm{mg} / \mathrm{kg}$. Quant au mercure et au zinc, leurs LMR relatives aux champignons ne sont pas encore fixées.

\section{Analyses statistiques}

Les statistiques descriptives et l'analyse de variance (ANOVA) des données ont été réalisées au moyen du logiciel de statistique XLSTAT 7.5.

Pour comparer les différentes espèces de champignon (T. robustus, T. letestui et $V$. volvacea) entre elles en tenant compte de la taille des échantillons, l'analyse de variance (ANOVA) à un facteur a été réalisée en utilisant «espèces de champignon » comme variable indépendante explicative et «teneurs 
métalliques » comme variable dépendante expliquée. Le niveau de confiance ou intervalle de confiance est $95 \%$, la probabilité de se tromper si l'on rejette l'hypothèse nulle (Ho) alors qu'elle est vraie, est donc $5 \%$. L'hypothèse nulle stipule qu'il n'y a pas de différence significative entre les espèces de champignon tandis que l'hypothèse alternative H1 stipule qu'il y a une différence significative entre les espèces de champignon. Le test utilisé pour les comparaisons multiples est celui de Fisher.

\section{RESULTATS ET DISCUSSION}

\section{Teneurs métalliques des champignons}

Les teneurs métalliques obtenues sont consignés dans les Tableaux 2 et 3 . La teneur minimale de chacun des trois métaux $(\mathrm{Pb}, \mathrm{Cd}$ et $\mathrm{Hg}$ ) est $0,01 \pm 0,01 \mathrm{mg} / \mathrm{kg}$ et celle du zinc est $0,001 \pm 0,001 \mathrm{mg} / \mathrm{kg}$. Quant à la teneur maximale, elle varie selon la nature du métal. Ainsi, la concentration maximale du plomb est de $1,11 \pm 0,01 \mathrm{mg} / \mathrm{kg}$ et a été détectée chez Termitomyces letestui. Concernant le cadmium, sa plus grande teneur qui est de $0,52 \pm 0,02 \mathrm{mg} / \mathrm{kg}$ a été mise en évidence chez Termitomyces robustus. Pour le mercure et le zinc, leurs teneurs maximales respectives sont $0,85 \pm 0,04 \mathrm{mg} / \mathrm{kg}$ et $0,07 \pm 0,03 \mathrm{mg} / \mathrm{kg}$ respectivement chez Termitomyces letestui et Termitomyces robustus.

Les teneurs métalliques moyennes de chaque espèce de champignon sont consignées dans le Tableau 4. Les teneurs moyennes des métaux oscillent entre $0,30 \pm 0,05 \mathrm{mg} / \mathrm{kg}(\mathrm{Pb})$ et $0,003 \pm 0,001 \mathrm{mg} / \mathrm{kg}(\mathrm{Zn})$.

Les teneurs métalliques (plomb et cadmium) de la présente étude sont très proches de celles qui ont été révélées par Radulescu et al. (2011). En effet, les études réalisées par ces auteurs ont montré la présence du plomb et du cadmium dans les champignons comestibles (Amanita caesarea, Pleurotus ostreatus, Fistulina hepatica et
Armillariella mellea), les concentrations métalliques variant de 0,25 à $1,89 \mathrm{mg} / \mathrm{kg}$ pour le plomb et, de 0,23 à $1,13 \mathrm{mg} / \mathrm{kg}$ pour le cadmium. Des concentrations similaires d'éléments traces métalliques (plomb et cadmium) ont été aussi trouvées par Konuk et al. (2006) dans 23 espèces de champignon sauvages comestibles. Ces teneurs sont 0,004 - 0,07 mg/kg (plomb) et 0,002 - 0,036 mg/kg (cadmium).

Par ailleurs, quelques auteurs ont trouvé des teneurs métalliques plus élevées que celles obtenues dans la présente étude. Ainsi, Zu et al. (2011) ont détecté du plomb, du cadmium et du zinc dans le Volvariella volvacea à des concentrations respectives de $1,61 \pm 0,09 \mathrm{mg} / \mathrm{kg}, 0,58 \pm 0,03 \mathrm{mg} / \mathrm{kg}$ et 66,2 $\pm 4,8 \mathrm{mg} / \mathrm{kg}$. Les autres espèces de champignons comestibles étudiés par $\mathrm{Zu}$ et al. (2011) ont des teneurs métalliques oscillant entre 0,06 - 0,58 mg/kg (cadmium), 0,67-12,9 $\mathrm{mg} / \mathrm{kg}$ (plomb) et 42,9-94,3 mg/kg (zinc). Durkan et al. (2008) ont également détecté $19,42 \mathrm{mg} / \mathrm{kg}$ de plomb dans le Stereum hirsutum, 202,1 mg/kg de zinc dans le Formes fomentarius et $0,663 \mathrm{mg} / \mathrm{kg}$ de cadmium dans le Suillus bellinui. Demirbas (2002) a aussi signalé $1,28 \mathrm{mg} / \mathrm{kg}$ de plomb, 2,48 $\mathrm{mg} / \mathrm{kg}$ de cadmium, $0,91 \mathrm{mg} / \mathrm{kg}$ de mercure et 76,8 $\mathrm{mg} / \mathrm{kg}$ de zinc dans l'Armillaria mellea, un champignon comestible.

\section{Evaluation du niveau de contamination des champignons}

Les teneurs métalliques moyennes de toutes les espèces de champignon sont inférieures aux limites maximales de résidus (LMR) du plomb $(0,3 \mathrm{mg} / \mathrm{kg})$ et du cadmium $(0,2 \mathrm{mg} / \mathrm{kg})$, fixées par l'Union Européenne (Figure 2). Etant donné que les teneurs moyennes du plomb et cadmium sont endessous des LMR, toutes ces espèces sont de bonne qualité par rapport à ces deux métaux. 
Cette conformité des teneurs du plomb et du cadmium à leurs LMR, a été aussi signalée par bon nombre d'auteurs, en l'occurrence Isildak et al. (2004) et Sarikurkcu et al. (2010). En effet, Isildak et al. (2004) ont détecté du plomb et cadmium dans des champignons cultivés (Marasmiuss oreades, Armillaria mellea, Morchella elata,Morchella vulgaris et Agaricus bisporus) à des teneurs en-dessous des LMR relatives à ces métaux. Quant à Sarikurkcu et al. (2010), leurs études ont permis de mettre en évidence l'Amanita caesarea, des teneurs de cadmium inférieures à la LMR.

La conformité des teneurs du plomb et du cadmium aux LMR, obtenue dans la présente étude, n'est pas toujours le cas dans la littérature car la plupart des études présentent les champignons comme d'excellents bioaccumulateurs des métaux et les teneurs en éléments traces métalliques des champignons excèdent parfois les limites maximales de résidus officielles. C'est le cas des travaux de Cocchi et al. (2006) où les teneurs du cadmium des espèces Amanita caesarea, Boletus edulis et Boletus pinophilus excèdent la limite maximale de résidu de ce métal et les teneurs du cadmium sont 50 fois plus élevées que la dose hebdomadaire de cadmium recommandée par l'organisation mondiale de la santé.

Le mercure et le zinc sont présents dans les trois espèces de champignons, cependant l'appréciation de la qualité de ces espèces de champignon par rapport à ces éléments traces métalliques n'a pu être faite par manque LMR officielles de ces deux métaux.

\section{Comparaison interspécifique des champignons}

Le zinc est le métal pour lequel le test de Fisher a révélé une différence significative entre Termitomyces robustus et Volvariella volvacea d'une part et d'autre part entre Termitomyces robustus et Termitomyces letestui car pour ces comparaisons, les pvalues sont respectives 0,022 et 0,046 sont inférieures à 5\%. Concernant les autres éléments traces métalliques (plomb, mercure et cadmium), il n'y a pas de différence significative entre les espèces de champignon étant donné que les p-values sont supérieures à $5 \%$. Ces p-values oscillent entre 0,083 et 0,979 (Tableau 6, 7, et 8).

Le Termitomyces robustus se démarque des deux autres espèces sur la base de leurs teneurs en zinc. Si l'on exclut la contamination que la manutention pourrait provoquer chez ces trois espèces fongiques, on peut dire que Termitomyces robustus accumule plus de zinc que le Volvariella. Volvacea et Termitomyces letestui.

Cette assertion est corroborée par les résultats de Radulescu et al. (2010) et d'Alonzo et al. (2003). En effet, Radulescu et al. (2010) ont observé une bioaccumulation du zinc par les champignons (Lycoperdon perlatum, Pleurotus ostreatus, Fistulina hepatica, Armillariella mellea), le coefficient de bioaccumulation se situant entre 1,01 et 2,01. Quant à Alonzo et al. (2003), ils ont montré que les teneurs d'éléments traces métalliques dépendent des espèces de champignon et que le zinc est fortement accumulé par Calvatia utriformis $(265,8$ $\mathrm{mg} / \mathrm{kg})$, Lactarius delicious $(231 \mathrm{mg} / \mathrm{kg})$, et Agaricus macrosporus (221,3 mg/kg).

Le mercure et le zinc sont présents dans les espèces de champignon étudiées, mais on ne peut porter un jugement de valeur sur les teneurs de ces métaux à cause du manque de valeur toxicologique de référence relative aux champignons comestibles. 
Tableau 1 : Limites de quantification et détection.

\begin{tabular}{lcc}
\hline ETM & $\begin{array}{c}\text { Limite de quantification }(\mathbf{L Q}) \\
(\mathbf{m g} / \mathbf{k g})\end{array}$ & $\begin{array}{c}\text { Limite de détection(LD) } \\
(\mathbf{m g} / \mathbf{k g})\end{array}$ \\
\hline Plomb & $8 \times 10^{-4}$ & $24 \times 10^{-6}$ \\
Cadmium & $8 \times 10^{-4}$ & $24 \times 10^{-6}$ \\
Mercure & $8 \times 10^{-5}$ & $8 \times 10-6$ \\
Zinc & 0,04 & $4 \times 10^{-4}$ \\
\hline
\end{tabular}

Tableau 2 : Teneurs métalliques de Termitomyces robustus.

\begin{tabular}{ccccc}
\hline \multicolumn{5}{c}{ Teneurs métalliques (mg/kg) } \\
\hline $\mathbf{N}^{\circ}$ Echantillon & $\mathbf{P b}$ & $\mathbf{C d}$ & $\mathbf{H g}$ & $\mathbf{Z n}$ \\
\hline 1 & $0,09 \pm 0,01$ & $<\mathrm{LQ}$ & $0,04 \pm 0,02$ & $<\mathrm{LQ}$ \\
2 & $0,09 \pm 0,05$ & $<\mathrm{LQ}$ & $0,25 \pm 0,14$ & 0,05 \\
3 & $0,22 \pm 0,08$ & $<\mathrm{LQ}$ & $0,25 \pm 0,08$ & 0,03 \\
4 & $0,04 \pm 0,01$ & $<\mathrm{LQ}$ & $0,28 \pm 0,09$ & $<\mathrm{LQ}$ \\
5 & $<\mathrm{LQ}$ & $<\mathrm{LQ}$ & $0,26 \pm 0,05$ & $<\mathrm{LQ}$ \\
6 & $0,01 \pm 0,01$ & $0,01 \pm 0,01$ & $0,25 \pm 0,04$ & $<\mathrm{LQ}$ \\
7 & $0,11 \pm 0,05$ & $<\mathrm{LQ}$ & $0,24 \pm 0,03$ & $0,01 \pm 0,01$ \\
8 & $0,26 \pm 0,09$ & $<\mathrm{LQ}$ & $0,30 \pm 0,07$ & $0,07 \pm 0,03$ \\
9 & $0,69 \pm 0,03$ & $<\mathrm{LQ}$ & $0,20 \pm 0,02$ & $0,02 \pm 0,01$ \\
10 & $0,86 \pm 0,1$ & $<\mathrm{LQ}$ & $<\mathrm{LQ}$ & $<\mathrm{LQ}$ \\
11 & $0,53 \pm 0,06$ & $<\mathrm{LQ}$ & $<\mathrm{LQ}$ & $<\mathrm{LQ}$ \\
12 & $0,37 \pm 0,03$ & $<\mathrm{LQ}$ & $<\mathrm{LQ}$ & $<\mathrm{LQ}$ \\
13 & $0,46 \pm 0,1$ & $<\mathrm{LQ}$ & $<\mathrm{LQ}$ & $<\mathrm{LQ}$ \\
14 & $0,35 \pm 0,08$ & $0,08 \pm 0,04$ & $<\mathrm{LQ}$ & $<\mathrm{LQ}$ \\
15 & $0,37 \pm 0,07$ & $0,01 \pm 0,01$ & $<\mathrm{LQ}$ & $<\mathrm{LQ}$ \\
16 & $0,21 \pm 0,02$ & $0,02 \pm 0,01$ & $<\mathrm{LQ}$ & $<\mathrm{LQ}$ \\
17 & $0,38 \pm 0,15$ & $0,52 \pm 0,02$ & $<\mathrm{LQ}$ & $<\mathrm{LQ}$ \\
\hline $\mathrm{LQ}$ & & & &
\end{tabular}

LQ : limite de quantification 
Tableau 3: Teneurs métalliques de Volvariella volvacea et de Termitomyces letestui.

\begin{tabular}{|c|c|c|c|c|c|c|c|c|}
\hline \multirow[b]{2}{*}{$\mathrm{N}^{\circ}$ echantillon } & \multicolumn{4}{|c|}{ Termitomyces letestui $(\mathrm{mg} / \mathrm{kg})$} & \multicolumn{4}{|c|}{ Volvariella volvacea $(\mathrm{mg} / \mathrm{kg})$} \\
\hline & $\mathbf{P b}$ & Cd & $\mathbf{H g}$ & $\mathbf{Z n}$ & $\mathbf{P b}$ & Cd & $\mathbf{H g}$ & $\mathbf{Z n}$ \\
\hline 1 & $0,11 \pm 0,08$ & $<\mathrm{LQ}$ & $<\mathrm{LQ}$ & $0,01 \pm 0,01$ & $0,03 \pm 0,01$ & $<\mathrm{LQ}$ & $0,01 \pm 0,01$ & $<\mathrm{LQ}$ \\
\hline 2 & $0,10 \pm 0,06$ & $<\mathrm{LQ}$ & $0,03 \pm 0,02$ & $<\mathrm{LQ}$ & $0,09 \pm 0,05$ & $<\mathrm{LQ}$ & $0,04 \pm 0,02$ & $0,01 \pm 0,01$ \\
\hline 3 & $0,05 \pm 0,02$ & $<\mathrm{LQ}$ & $0,04 \pm 0,02$ & $<\mathrm{LQ}$ & $0,13 \pm 0,08$ & $<\mathrm{LQ}$ & $0,05 \pm 0,03$ & $<\mathrm{LQ}$ \\
\hline 4 & $0,07 \pm 0,03$ & $<\mathrm{LQ}$ & $<\mathrm{LQ}$ & $0,01 \pm 0,01$ & $0,01 \pm 0,01$ & $<\mathrm{LQ}$ & $0,05 \pm 0,04$ & $<\mathrm{LQ}$ \\
\hline 5 & $0,07 \pm 0,04$ & $<\mathrm{LQ}$ & $0,04 \pm 0,02$ & $<\mathrm{LQ}$ & $0,07 \pm 0,02$ & $<\mathrm{LQ}$ & $0,05 \pm 0,03$ & $0,01 \pm 0,01$ \\
\hline 6 & $0,06 \pm 0,02$ & $<\mathrm{LQ}$ & $0,05 \pm 0,03$ & $<\mathrm{LQ}$ & $0,05 \pm 0,03$ & $<\mathrm{LQ}$ & $0,04 \pm 0,02$ & $0,01 \pm 0,02$ \\
\hline 7 & $0,10 \pm 0,06$ & $0,01 \pm 0,01$ & $0,03 \pm 0,01$ & $0,02 \pm 0,01$ & $0,13 \pm 0,05$ & $<\mathrm{LQ}$ & $0,08 \pm 0,01$ & $0,01 \pm 0,01$ \\
\hline 8 & $0,01 \pm 0,01$ & $<\mathrm{LQ}$ & $0,08 \pm 0,04$ & $0,01 \pm 0,01$ & $0,05 \pm 0,03$ & $<\mathrm{LQ}$ & $0,04 \pm 0,01$ & $0,01 \pm 0,01$ \\
\hline 9 & $0,03 \pm 0,01$ & $<\mathrm{LQ}$ & $0,06 \pm 0,04$ & $0,01 \pm 0,01$ & $0,10 \pm 0,08$ & $<\mathrm{LQ}$ & $0,05 \pm 0,01$ & $<\mathrm{LQ}$ \\
\hline 10 & $<\mathrm{LQ}$ & $0,02 \pm 0,01$ & $0,09 \pm 0,03$ & $<\mathrm{LQ}$ & $0,02 \pm 0,01$ & $0,07 \pm 0,03$ & $0,03 \pm 0,02$ & $0,01 \pm 0,01$ \\
\hline 11 & $<\mathrm{LQ}$ & $0,02 \pm 0,01$ & $0,11 \pm 0,07$ & $<\mathrm{LQ}$ & $<\mathrm{LQ}$ & $0,02 \pm 0,01$ & $0,04 \pm 0,03$ & $0,01 \pm 0,01$ \\
\hline 12 & $<\mathrm{LQ}$ & $0,01 \pm 0,01$ & $0,07 \pm 0,03$ & $<\mathrm{LQ}$ & $<\mathrm{LQ}$ & $0,01 \pm 0,01$ & $0,06 \pm 0,04$ & $0,01 \pm 0,01$ \\
\hline 13 & $<\mathrm{LQ}$ & $0,02 \pm 0,01$ & $0,07 \pm 0,03$ & $<\mathrm{LQ}$ & $0,21 \pm 0,16$ & $<\mathrm{LQ}$ & $<\mathrm{LQ}$ & $<\mathrm{LQ}$ \\
\hline 14 & $0,12 \pm 0,05$ & $0,01 \pm 0,01$ & $0,10 \pm 0,05$ & $<\mathrm{LQ}$ & $0,58 \pm 0,04$ & $<\mathrm{LQ}$ & $0,01 \pm 0,01$ & $<\mathrm{LQ}$ \\
\hline 15 & $0,14 \pm 0,04$ & $<\mathrm{LQ}$ & $0,11 \pm 0,05$ & $<\mathrm{LQ}$ & $0,21 \pm 0,03$ & $0,34 \pm 0,06$ & $0,01 \pm 0,01$ & $<\mathrm{LQ}$ \\
\hline 16 & $0,25 \pm 0,09$ & $0,03 \pm 0,02$ & $0,10 \pm 0,04$ & $<\mathrm{LQ}$ & $0,04 \pm 0,01$ & $<\mathrm{LQ}$ & $0,54 \pm 0,2$ & $<\mathrm{LQ}$ \\
\hline 17 & $0,06 \pm 0,02$ & $0,02 \pm 0,01$ & $0,10 \pm 0,03$ & $<\mathrm{LQ}$ & $0,43 \pm 0,03$ & $<\mathrm{LQ}$ & $0,03 \pm 0,01$ & $<\mathrm{LQ}$ \\
\hline 18 & $0,14 \pm 0,06$ & $0,01 \pm 0,01$ & $0,13 \pm 0,06$ & $<\mathrm{LQ}$ & $0,57 \pm 0,22$ & $<\mathrm{LQ}$ & $0,04 \pm 0,02$ & $<\mathrm{LQ}$ \\
\hline 19 & $0,33 \pm 0,11$ & $<\mathrm{LQ}$ & $<\mathrm{LQ}$ & $0,01 \pm 0,01$ & $0,45 \pm 0,17$ & $<\mathrm{LQ}$ & $0,05 \pm 0,03$ & $<\mathrm{LQ}$ \\
\hline 20 & $0,06 \pm 0,02$ & $0,01 \pm 0,01$ & $<\mathrm{LQ}$ & 0,01 & $0,43 \pm 0,10$ & $0,08 \pm 0,04$ & $0,32 \pm 0,22$ & $0,01 \pm 0,01$ \\
\hline 21 & $1,11 \pm 0,13$ & $<\mathrm{LQ}$ & $<\mathrm{LQ}$ & $<\mathrm{LQ}$ & $0,48 \pm 0,20$ & $0,01 \pm 0,01$ & $0,03 \pm 0,02$ & $<\mathrm{LQ}$ \\
\hline 22 & $<\mathrm{LQ}$ & $0,01 \pm 0,01$ & $<\mathrm{LQ}$ & $<\mathrm{LQ}$ & $0,53 \pm 0,13$ & $0,25 \pm 0,08$ & $0,04 \pm 0,02$ & $<\mathrm{LQ}$ \\
\hline 23 & $<\mathrm{LQ}$ & $0,01 \pm 0,01$ & $<\mathrm{LQ}$ & $<\mathrm{LQ}$ & $0,48 \pm 0,21$ & $<\mathrm{LQ}$ & $0,06 \pm 0,04$ & $<\mathrm{LQ}$ \\
\hline 24 & $0,73 \pm 0,20$ & $<\mathrm{LQ}$ & $<\mathrm{LQ}$ & $0,01 \pm 0,01$ & $0,42 \pm 0,03$ & $0,12 \pm 0,07$ & $<\mathrm{LQ}$ & $<\mathrm{LQ}$ \\
\hline 25 & $0,74 \pm 0,32$ & $0,01 \pm 0,01$ & $<\mathrm{LQ}$ & $0,01 \pm 0,01$ & $0,33 \pm 0,07$ & $<$ LQ & $<\mathrm{LQ}$ & $<\mathrm{LQ}$ \\
\hline 26 & $0,50 \pm 0,02$ & $<\mathrm{LQ}$ & $<\mathrm{LQ}$ & $<\mathrm{LQ}$ & $0,36 \pm 0,08$ & $<\mathrm{LQ}$ & $<\mathrm{LQ}$ & $<\mathrm{LQ}$ \\
\hline 27 & $<\mathrm{LQ}$ & $0,01 \pm 0,01$ & $0,85 \pm 0,04$ & $<\mathrm{LQ}$ & $0,14 \pm 0,06$ & $0,08 \pm 0,04$ & $<\mathrm{LQ}$ & $<\mathrm{LQ}$ \\
\hline 28 & $0,19 \pm 0,07$ & $0,01 \pm 0,01$ & $0,77 \pm 0,03$ & $<\mathrm{LQ}$ & $0,43 \pm 0,05$ & $<\mathrm{LQ}$ & $0,34 \pm 0,18$ & $0,01 \pm 0,01$ \\
\hline 29 & $0,10 \pm 0,04$ & $0,01 \pm 0,01$ & $0,76 \pm 0,05$ & $0,01 \pm 0,01$ & $0,52 \pm 0,02$ & $0,01 \pm 0,01$ & $0,28 \pm 0,11$ & $<\mathrm{LQ}$ \\
\hline 30 & $0,02 \pm 0,01$ & $<\mathrm{LQ}$ & $0,02 \pm 0,01$ & $0,01 \pm 0,01$ & $0,44 \pm 0,03$ & $<$ LQ & $0,29 \pm 0,17$ & $<\mathrm{LQ}$ \\
\hline
\end{tabular}


Tableau 4: Statistiques descriptives des teneurs métalliques des champignons.

\begin{tabular}{lccccccc}
\hline Espèces & métaux & Min & $\mathbf{1}^{\text {er }}$ quartile & Médiane & $\mathbf{3}^{\text {è }}$ quartile & Max & Moy \\
\hline \multirow{3}{*}{ T. robustus } & $\mathrm{Pb}$ & 0,01 & 0,09 & 0,26 & 0,42 & 0,86 & 0,30 \\
& $\mathrm{Cd}$ & 0,01 & 0,0 & 0,0 & 0,01 & 0,52 & 0,04 \\
& $\mathrm{Hg}$ & 0,01 & 0,0 & 0,04 & 0,25 & 0,30 & 0,12 \\
& $\mathrm{Zn}$ & 0,01 & 0,0 & 0,0 & 0,10 & 0,07 & 0,01 \\
\hline \multirow{3}{*}{ letestui } & $\mathrm{Pb}$ & 0,01 & 0,01 & 0,07 & 0,14 & 1,11 & 0,17 \\
& $\mathrm{Cd}$ & 0,01 & 0,00 & 0,01 & 0,01 & 0,03 & 0,01 \\
& $\mathrm{Hg}$ & 0,01 & 0,00 & 0,045 & 0,10 & 0,85 & 0,12 \\
& $\mathrm{Zn}$ & 0,01 & 0,00 & 0,00 & 0,01 & 0,02 & 0,004 \\
\hline \multirow{3}{*}{ volvacea } & $\mathrm{Pb}$ & 0,01 & 0,05 & 0,21 & 0,44 & 0,58 & 0,26 \\
& $\mathrm{Cd}$ & 0,01 & 0,00 & 0,00 & 0,01 & 0,34 & 0,03 \\
& $\mathrm{Hg}$ & 0,01 & 0,01 & 0,04 & 0,06 & 0,54 & 0,09 \\
& $\mathrm{Zn}$ & 0,001 & 0,00 & 0,00 & 0,01 & 0,01 & 0,003 \\
\hline
\end{tabular}

Min : minimum ; Moy : moyenne ; Max : maximum.

Tableau 5 : Test de Fisher pour le zinc: Analyse des différences entre les groupes avec un intervalle de confiance à $95,00 \%$.

\begin{tabular}{lccccc}
\hline Modalités & Différence & $\begin{array}{c}\text { Différence } \\
\text { réduite }\end{array}$ & $\begin{array}{c}\text { Valeur } \\
\text { critique }\end{array}$ & Pr. > Diff & Significatif \\
\hline T. robustus - V. volvacea & 0,008 & 2,340 & 1,993 & 0,022 & Oui \\
T. robustus - T. letestui & 0,007 & 2,032 & 1,993 & 0,046 & Oui \\
T. letestui $-V$. volvacea & 0,001 & 0,363 & 1,993 & 0,718 & Non \\
\hline
\end{tabular}

Tableau 6: Test de Fisher pour le plomb: Analyse des différences entre les groupes avec un intervalle de confiance à $95,00 \%$.

\begin{tabular}{lccccc}
\hline Modalités & Différence & $\begin{array}{c}\text { Différence } \\
\text { réduite }\end{array}$ & $\begin{array}{c}\text { Valeur } \\
\text { critique }\end{array}$ & Pr. > Diff & Significatif \\
\hline T. robustus - T. letestui & 0,127 & 1,755 & 1,993 & 0,083 & Non \\
T. robustus - V. volvacea & 0,039 & 0,537 & 1,993 & 0,593 & Non \\
V. volvacea - T. letestui & 0,088 & 1,432 & 1,993 & 0,156 & Non \\
\hline
\end{tabular}

Tableau 7: Test de Fisher pour le cadmium : Analyse des différences entre les groupes avec un intervalle de confiance à $95,00 \%$.

\begin{tabular}{lccccc}
\hline Modalités & Différence & $\begin{array}{c}\text { Différence } \\
\text { réduite }\end{array}$ & $\begin{array}{c}\text { Valeur } \\
\text { critique }\end{array}$ & Pr. > Diff & Significatif \\
\hline T. robustus - T. letestui & 0,030 & 1,305 & 1,993 & 0,196 & Non \\
T. robustus $-V$. volvacea & 0,005 & 0,200 & 1,993 & 0,842 & Non \\
V. volvacea - T. letestui & 0,026 & 1,299 & 1,993 & 0,198 & Non \\
\hline
\end{tabular}


Tableau 8: Test de Fisher pour le mercure : Analyse des différences entre les groupes avec un intervalle de confiance à $95,00 \%$.

\begin{tabular}{lccccc}
\hline Modalités & Différence & $\begin{array}{c}\text { Différence } \\
\text { réduite }\end{array}$ & $\begin{array}{c}\text { Valeur } \\
\text { critique }\end{array}$ & $\begin{array}{c}\text { Pr. }> \\
\text { Diff }\end{array}$ & Significatif \\
\hline T. robustus - T. letestui & 0,036 & 0,665 & 1,993 & 0,508 & Non \\
T. robustus - V. volvacea & 0,001 & 0,027 & 1,993 & 0,979 & Non \\
V. volvacea - T. letestui & 0,034 & 0,750 & 1,993 & 0,456 & Non \\
\hline
\end{tabular}



Figure 1: Courbe d'étalonnage du plomb.

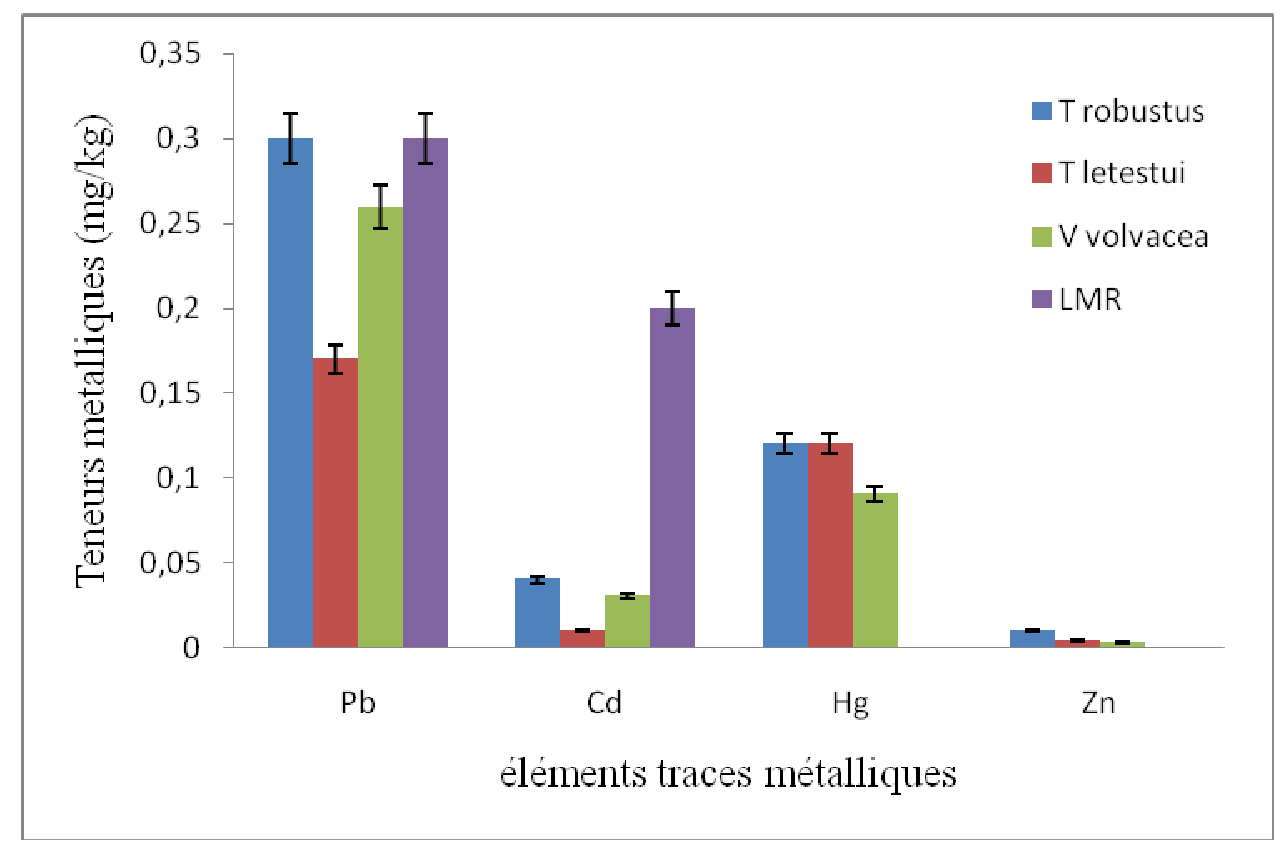

Figure 2: Histogramme comparative des teneurs moyennes du plomb et du cadmium aux LMR. 


\section{Conclusion}

Volvariella volvacea, Termitomyces robustus et Termitomyces letestui rencontrés sur les marchés Abidjanais ne sont pas épargnés par les éléments traces métalliques plomb, cadmium, mercure et zinc. Les teneurs moyennes de plomb et de cadmium sont inférieures à leurs normes respectives fixées par le règlement $n^{\circ}$ 466/2001 de la Commission Européenne. Les concentrations métalliques des trois espèces ( $V$. volvacea, $T$. robustus et $T$. letestui) sont basses par rapport à celles révélées par la plupart des études effectuées ailleurs dans le monde. Cependant, certains échantillons de champignon sont fortement contaminés par les éléments traces métalliques (teneurs métalliques supérieures aux LMR).

La consommation de ces espèces de champignon doit être modérée, car ce matériel biologique pourrait contribuer à l'augmentation de la quantité d'éléments traces métalliques dans l'organisme.

\section{REMERCIEMENTS}

Nous remercions le Laboratoire Central d'Agrochimie et d'Ecotoxicologie (LCAE), le Laboratoire Central pour l'Hygiène Alimentaire et de l'Agro-Industrie (LCHAI) pour leur importante contribution à la réalisation de ce travail. Nos remerciements sont vivement adressés aux personnels de ces deux laboratoires pour leur disponibilité et leur esprit d'équipe.

\section{REFERENCES}

Alonso J, Garcia MA, Perez-Lopez M, Melgar MJ. 2003. The concentrations and bioconcentration factors of copper and zinc in edible mushrooms. Archives of Environmental Contamination and Toxicology, 44(2): 180188.

AOAC. 2003. Official method 999. Lead, cadmium, zinc, copper and iron in foods. AOAC.

AOAC. 1978. Official method 977.13. Mercury in fish. AOAC.

Athanasakis G, Aligiannis N, Gonou-Zagou Z, Skaltsounis AL, Fokialakis N. 2013. Antioxidant properties of the wild edible mushroom Lactarius salmonicolor. Journal of Medicinal Food, 16(8): 760-76.
Cocchi L, Vescovi L, Petrini LE, Petrin O. Heavy metals in edible mushrooms in Italy. Food Chemistry, 98(2): 277-284.

Demirbas A. 2002. Metal ion uptake by mushrooms from natural and artificially enriched soil. Food Chemistry, 1(78): 89-93.

Durkan N, Isiloglu M, Kabar K, Dogan Y. 2008. Heavy metal levels in some macrofungi from Menderes river basin, Turkey. Natura Monitenegrina, Podgorica, 7(2): 465-473.

Györfi J, Geösel A, Vetter J. 2010. Mineral composition of different strains of edible medicinal Mushroom Agaricus subrufescens Peck. Journal of Medicinal Food, 13(6): 15101514.

Isildak O, Turkekul I, Elmastas M, Tuzen M. 2005. Analysis of heavy metals in some wildgrown edible mushrooms from the middle black sea region, Turkey. Food Chemistry, 86(4): 547-552.

Mathialagan T, Viraraghavan T, Cullimore DR. 2003. Adsorption of cadmium from aqueous solutions by edible mushrooms (Agaricus bisporusand, Lentinus edodes). Water Quality Research Journal of Canada, 38(3): 499-514.

Konuk M, Afyonand A, Yagız D.2007. Minor element and heavy metal contents of wild growing and edible mushrooms from western black sea region of turkey. Fresenius Environmental Bulletin, 16(11): 1359-1362.

Mujic I, Zekovic Z, Vidovic S, Radojkovic M, Zivkovic J, Go Cevac D. 2011. Fatty acid profiles of four wild mushrooms and their potential benefits for hypertension treatment. Journal of Medicinal Food, 14(11): 1330-1337.

Musa B, Abdullahi MS. 2013. The toxicological effects of cadmium and some other heavy metals in plants and humans. Journal of Environmental Science and Water Resources, 2(8): 245-249.

Nassif N, Saade Z. 2010. Studying heavy metals in sediments layers along selected sites on the lebanese coast. Journal of Water Resource and Protection, 2(1): 48-60.

Radulescu C, Stihi C, Busuioc G, Gheboianu AI, Popescu IV. 2010. Studies concerning heavy metals bioaccumulation of wild edible mushrooms from industrial area by using spectrometric techniques. Bulletin of 
Environmental Contamination and Toxicology, 84(5): 641-646.

Radulescu C, Stihi C, Cimpoca VG, Popescu IV, Busuioc G, Gheboianu AI. 2011. Evaluation of heavy metals content in edible mushrooms by microwave digestion and flame atomic absorption spectrometry. Scientific Study \& Research, 12(2): 155-164.

Règlement CE. 2006. Règlement (CE) n 1881/2006 de la Commission du 19 décembre 2006 portant fixation des teneurs maximales pour certains contaminants dans les denrées alimentaires.

Sarikurkcu C, Tepe B, Semiz DK, Solak MH. 2010. Evaluation of metal concentration and antioxidant activity of three edible mushrooms from Mugla, Turkey. Food and Chemical Toxicology, 48(5): 1230-1233.

Shivakumar D, Srikantaswamy S, Sreenivasa S, Kiran BM. 2012. Speciation and geochemical behaviour of heavy metals in industrial area soil of Mysore City, India. Journal of Environmental Protection, 3(10): 1384-1392.

Soares R, Meireles M, Rocha A, Pirraco A, Obiol D, Alonso E, Joos G, Balogh G. 2011. Maitake (D fraction) mushroom extract induces apoptosis in breast cancer cells by BAK-1gene activation. Journal of Medicinal Food, 14(6): 563-572.

Unak P, Lambrecht FY, Biber FZ, Darcan S. 2007. Iodine measurements by isotope dilution analysis in drinking water in Western Turkey. Journal of Radioanalytical and Nuclear Chemistry, 273(3): 649-651.

Zhu F, Qu L, Fan W, Qiao M, Hao H, Wan X. 2011. Assessment of heavy metals in some wild edible mushrooms collected from Yunnan Province, China. Environmental Monitoring and Assessment, 179(1-4): 191-199. 